\title{
ON MINIMAL POTENTIALLY POWER-POSITIVE SIGN PATTERNS
}

\author{
BER-LIN Yu AND TING-ZHU HUANG
}

Abstract. An $n$-by- $n$ sign pattern $\mathscr{A}$ is said to be potentially power-positive if there exists some $A \in Q(\mathscr{A})$ such that $A$ is power-positive, i.e., $A^{k}>0$ for some positive integer $k$. Catral, Hogben, Olesky and van den Driessche [Sign patterns that require or allow power-positivity, Electron. J. Linear Algebra, 19 (2010), 121-128] investigated the sign patterns that require or allow power-positivity. It has been shown that an $n$-by- $n$ sign pattern $\mathscr{A}$ is potentially power-positive if and only if either $\mathscr{A}$ or $-\mathscr{A}$ is potentially eventually positive. But as the identification of sufficient and necessary conditions for potentially eventually positive sign patterns remains open, the characterization of potentially power-positive sign patterns is still open. In this paper, we introduce the minimal potentially power-positive sign patterns to classify the potentially powerpositive sign patterns. Some properties of minimal potentially power-positive sign patterns are presented. It is shown that for an $n$-by- $n$ sign pattern $\mathscr{A}$ with at most $n+1$ negative entries, $\mathscr{A}$ is minimal potentially power-positive if and only if either $\mathscr{A}$ or $-\mathscr{A}$ is minimal potentially eventually positive. Finally, we classify the minimal potentially power-positive sign patterns of order $n \leqslant 3$.

Mathematics subject classification (2010): 15B35, 05 C50.

Keywords and phrases: Potentially eventually positive sign pattern, potentially power-positive sign pattern, minimal potentially power-positive sign pattern, irreducible pattern, primitive digraph.

\section{REFERENCES}

[1] A. Berman, M. Catral, L. M. DeAlba, A. Elhashash, F. J. Hall, L. Hogben, I.-J. Kim, D. D. Olesky, P. TARaZaga, M. J. Ts atsomeros and P. VAn Den Driessche, Sign patterns that allow eventual positivity, Electron. J. Linear Algebra 12 (2010), 108-120.

[2] A. Berman And R. J. Plemmons, Nonnegative Matrices in the Mathematical Sciences, SIAM, Philadelphia, 1994.

[3] A. BRAUER, On the characteristic roots of power-positive matrices, Duke Math. J. 28 (1961), 439445 .

[4] R. A. Brualdi and H. J. Ryser, Combinatorial Matrix Theory, Cambridge University Press, Cambridge, 1991.

[5] M. Catral, L. Hogben, D. D. Olesk y and P. van Den Driessche, Sign patterns that require or allow power-positivity, Electron. J. Linear Algebra 19 (2010), 121-128.

[6] E. M. Ellison, L. HogBen AND M. J. TSATSOMERos, Sign patterns that require eventual positivity or require eventual nonnegativity, Electron. J. Linear Algebra 19 (2010), 98-107.

[7] C. A. EsChenBACH AND C. R. Johnson, Sign patterns that require real, nonreal and pure imaginary eigenvalues, Linear and Multilinear Algebra 29 (1991), 299-311.

[8] Y. GaO, Z. Li AND Y. ShaO, Sign patterns allowing nilpotence of index 3, Linear Algebra Appl. 424 (2007), 55-70.

[9] F. J. Hall ANd Z. Li, Sign pattern matrices, in: L. Hogben(Ed.), Handbook of Linear Algebra, Chapman \& Hall/CRC Press, Boca Ration, 2007.

[10] F. J. HALl, Z. Li AND D. WANG, Symmetric sign pattern matrices that require unique inertina, Linear Algebra Appl. 338 (2001), 153-169.

[11] R. A. Horn And C. R. Johnson, Matrix Analysis, Cambridge University Press, New York, 1995.

[12] D. Noutsos, On Perron-Frobenius property of matrices having some negative entries, Linear Aglebra Appl. 412 (2006), 132-153. 